\title{
Lipid and fatty acid analysis of fresh and frozen-thawed immature and in vitro matured bovine oocytes
}

\author{
J. Y. Kim ${ }^{1}$, M. Kinoshita ${ }^{2}$, M. Ohnishi ${ }^{2}$ and Y. Fukui ${ }^{1 *}$ \\ ${ }^{1}$ Laboratory of Animal Genetics and Reproduction and ${ }^{2}$ Laboratory of Applied Bioscience, \\ Obihiro University of Agriculture and Veterinary Medicine, Obihiro 080-8555, Japan
}

The lipid content and fatty acid composition of fresh immature and in vitro matured bovine oocytes cultured in media with or without serum, and also those of frozen-thawed immature oocytes were analysed. All oocytes were ranked (A or B) on the basis of their cytoplasmic quality. Fatty acid composition ( $\mathrm{mol} \% ; \mathrm{w} / \mathrm{w}$ ) in the total lipid fraction was analysed by gas chromatography. Triglyceride, total cholesterol, phospholipid (phosphocholine-containing phospholipid) and non-esterified fatty acid contents of immature and in vitro matured oocytes were determined using lipid analysis kits. Phosphocholine-containing phospholipid and non-esterified fatty acid contents were determined in frozen-thawed immature bovine oocytes. Palmitic acid was the most abundant fatty acid in immature oocytes (A: 35\%, B: 36\%), and in in vitro matured oocytes cultured in the medium containing serum (A: 36\%, B: 35\%) or polyvinyl alcohol (A: $33 \%$, B: $36 \%$ ). Oleic acid was the second most abundant fatty acid in all A ranked oocytes, whereas stearic acid was the second most abundant fatty acid in all $B$ ranked oocytes. There were significant differences $(P<0.05)$ in linoleic and arachidonic acid fractions between A and B ranked immature oocytes. In vitro matured oocytes had significantly $(P<0.05)$ lower proportions of linoleic and arachidonic acids, and significantly $(P<0.01)$ lower contents of triglyceride and total cholesterol compared with those of immature oocytes. The fatty acid composition of in vitro matured oocytes cultured in medium containing fetal calf serum or polyvinyl alcohol was similar, but significant differences $(P<0.01)$ in triglyceride and the total cholesterol content were observed. There was a significant decrease $(P<0.05)$ in the arachidonic acid proportion in frozen-thawed immature oocytes compared with that in fresh immature oocytes. In addition, significant $(P<0.05)$ decreases in both phospholipid (15.8-10.6 pmol) and non-esterified fatty acid (11.0-4.1 pmol) were found in frozen-thawed immature oocytes. The results indicate that lipids are available for use as an energy source for maturation and that serum lipids are incorporated into the oocyte cytoplasm during in vitro maturation. The changes in the lipid content (mainly phospholipid) and fatty acid composition were also observed in frozen-thawed immature oocytes. The study indicates that the alteration of fatty acid composition in bovine oocytes might improve maturation and cryopreservation.

\section{Introduction}

Lipid plays a significant role in energy storage, cell structure and in modifying the physical properties and metabolic function of biological membranes. Studies on lipids in bovine oocytes and embryos have examined the fatty acid composition and triglyceride content (Ferguson and Leese, 1999; Zeron et al., 1999a; McEvoy et al., 2000). Other reports have indicated that intracellular lipid may have an important influence on sensitivity to freezing (Diez et al., 1996; Dobrinsky, 1996; Otoi et al., 1997; Sata et al., 1999). However, in terms of qualitative and quantitative analysis of fatty acid and lipid content, data on bovine oocytes are still limited. In particular, it has not been established whether storage and membrane lipids influence oocyte maturation and cryopreservation.

*Correspondence

Email: fukui@obihiro.ac.jp
Oocytes of all species are sensitive to low temperatures (Leibo et al., 1995). For several decades, limited success has been achieved after thawing of cryopreserved bovine oocytes. It has been reported that the cytoplasmic membrane is the major organelle sensitive to low temperature (Schmidt et al., 1993; Arav et al., 1996). The physical properties of this membrane are influenced by lipid composition and are regulated in response to environmental factors (temperature or diet) (Gurr and Harwood, 1991). Thus, understanding of the involvement of cytoplasmic and membrane lipids in oocyte sensitivity to low temperature and freezing damage is required to address the difficulties of development after cryopreservation of oocytes.

Serum is a common constituent of culture media. Although serum can be a useful source of growth factors and other cellular mitogenic factors, there are some disadvantages to its use. In ruminant embryos developed in a serum-supplemented medium, morphological differences, such as abundant cytoplasmic lipid droplets, have been observed (Thompson et al., 1995; Abe et al., 1999). Several 
studies have focused on the influence of serum in view of lipid accumulation and the changes in the fatty acid composition in oocytes and embryos (Ferguson and Leese 1999; Sata et al., 1999). Familiarity with the lipids present in serum and with the mechanism for transfer of these lipids to the cells is necessary to understand the mechanism involved in the lipid nutrition of cells in culture (Spector, 1972). Furthermore, detailed studies on morphological characteristics of oocytes are required to provide workable criteria for the selection of the best quality oocytes for in vitro production of bovine embryos.

The aim of the present study was to measure quantitatively the lipid content and fatty acid composition in fresh immature and in vitro matured bovine oocytes cultured in media with or without serum, and the lipid content (mainly phospholipid) and fatty acid composition in frozen-thawed immature oocytes.

\section{Materials and Methods}

\section{Collection of oocytes and sample preparation}

Ovaries were collected from Holstein cows at a local abattoir and transported within $3 \mathrm{~h}$ to the laboratory in $0.9 \%$ (w/v) $\mathrm{NaCl}$ at $30-35^{\circ} \mathrm{C}$. Cumulus-oocytes complexes (COCs) were collected by aspirating follicles of $2-8 \mathrm{~mm}$ in diameter with an 18-gauge needle followed by three washes in Dulbecco's PBS (Sigma, St Louis, MO). COCs were selected and classified as A or B using the criteria of de Loos et al. (1989). COCs ranked as $A$, equivalent to category 1 of the criteria described by de Loos et al. (1989), had homogeneous ooplasm and compact multi-layered cumulus cells tightly adherent to the zona pellucida. COCs ranked as B, combining categories 2 and 3 of the criteria of de Loos et al. (1989), were of coarse appearance with a dark zone at the periphery of the oocytes, but also had multi-layered cumulus cells tightly adherent to the zona pellucida. COCs ranked as A or B were assigned for freezing and thawing, and in vitro maturation, or were prepared immediately for lipid extraction. COCs were denuded by vigorous pipetting in four-well dishes (NUNCLON; Nunc, Roskilde) with $500 \mu \mathrm{l}$ of $1 \%$ (w/v) sodium citrate. The denuded immature oocytes were washed three times in $500 \mu \mathrm{l}$ PBS with $0.3 \%$ BSA (essentially fatty acid free; Sigma) with repeated gentle pipetting. Oocytes were transferred to a Petri dish containing $3 \mathrm{ml} \mathrm{PBS}$ with $0.1 \%$ BSA for reselection, and were washed three times. Finally, oocytes were washed twice in PBS without BSA. Subsequently, 250-400 fully denuded oocytes with intact zonae pellucidae were centrifuged at $4000 \mathrm{~g}$ for $5 \mathrm{~min}$ and resuspended by vortexing in $500 \mu \mathrm{l}$ PBS. Oocytes were transferred into a glass vial with $1.0 \mathrm{ml}$ PBS for total lipid extraction.

\section{Freezing and thawing}

COCs were frozen-thawed using techniques reported by Asada et al. (2000). Dulbecco's PBS containing 10\% (v/v) fetal calf serum (FCS), $1.8 \mathrm{~mol}$ ethylene glycol $\mathrm{I}^{-1}$ and 0.1 mol sucrose $\mathrm{I}^{-1}$ was used as the cryoprotective medium.
Ranked COCs were transferred to a cryoprotective medium and were equilibrated for $10 \mathrm{~min}$ at room temperature $\left(20-25^{\circ} \mathrm{C}\right)$. During the equilibration, groups of 20-25 COCs were loaded into $0.25 \mathrm{ml}$ straws (Fujihira Industry, Co., Tokyo), powder-sealed and transferred into a pre-cooled methanol bath $\left(0^{\circ} \mathrm{C}\right)$ of a programmable freezer $(\mathrm{ET}-1$, Fujihira). The straws were cooled from $0^{\circ} \mathrm{C}$ to $-6^{\circ} \mathrm{C}$ at $0.5^{\circ} \mathrm{C}$ per min. At $-6^{\circ} \mathrm{C}$, ice formation (seeding) was induced in the medium by touching the straw with frozen forceps. After 12 min, cooling was resumed at $0.3{ }^{\circ} \mathrm{C} \mathrm{min}^{-1}$ to $-35^{\circ} \mathrm{C}$. The straws were placed in liquid nitrogen vapour for $10 \mathrm{~s}$ and immersed in liquid nitrogen. Before lipid analysis, straws were thawed by exposure to the air for $10 \mathrm{~s}$ at $25^{\circ} \mathrm{C}$ and immersed immediately in water at $37^{\circ} \mathrm{C}$ for $20 \mathrm{~s}$. The COCs were denuded and washed three times in PBS containing $0.3 \%$ BSA. Intact oocytes with no discoloration of the ooplasm, cell disruption or pyknosis or those that had a dark ooplasm were selected according to the criteria described by Al Hasani and Diedrich (1995). Selected frozen-thawed oocytes were washed twice in PBS containing $0.1 \%$ BSA and then washed in PBS without BSA. Oocytes were subsequently treated for lipid extraction in the same manner as fresh immature oocytes.

\section{In vitro maturation}

COCs were matured in vitro using procedures modified from those of Lee and Fukui (1995). Briefly, COCs were cultured for $24 \mathrm{~h}$ in four-well dishes containing tissue culture medium 199 (TCM-199 with Earle's salts and glutamine; ICN Biomedicals, Inc., Costa, CA) supplemented with either $10 \%$ heat-inactivated FCS or $0.1 \%(\mathrm{w} / \mathrm{v})$ polyvinyl alcohol, $1 \mu \mathrm{g}$ oestradiol $\mathrm{ml}^{-1}$ (Sigma) and $0.02 \mathrm{AU}$ $\mathrm{FSH} \mathrm{ml} \mathrm{m}^{-1}$ (Antrin, Denka Chemical Co., Ltd, Kawasaki) (35-40 COCs per well), at $39^{\circ} \mathrm{C}$ in $5 \% \mathrm{CO}_{2}$ and $95 \%$ humidified air.

After maturation, the COCs were denuded and washed as described for immature oocytes. The oocytes were examined using a microscope to determine the stage of maturation. Only those oocytes that had extruded the first polar body were selected and prepared for total lipid extraction.

\section{Total lipid extraction}

Total lipids of fresh and frozen-thawed immature bovine oocytes and those of in vitro matured oocytes were extracted using the procedure of Bligh and Dyer (1959), with a slight modification. The samples $(n=250-400)$, suspended in $1.0 \mathrm{ml}$ PBS, were added immediately to $3.75 \mathrm{ml}$ methanol:chloroform $(1: 2 ; \mathrm{v} / \mathrm{v})$ containing $0.002 \%$ $(\mathrm{w} / \mathrm{v})$ butylated hydroxytoluene (to inhibit free radicalinitiated oxidation reactions) and sonicated for $5 \mathrm{~min}$. After sonication, chloroform $(1.25 \mathrm{ml})$ and water $(1.25 \mathrm{ml})$ were added to the mixture, which was centrifuged at $1500 \mathrm{~g}$ for $10 \mathrm{~min}$. The lower chloroform layer was transferred to a new tube and $2 \mathrm{ml}$ methanol:water (10:9; v/v) was added. The samples were mixed vigorously and centrifuged at 
$1500 \mathrm{~g}$ for $10 \mathrm{~min}$. The lower chloroform layer was withdrawn and evaporated to dryness under nitrogen. The residues (total lipids) were dissolved into chloroform:methanol (2:1; $\mathrm{v} / \mathrm{v})$ and stored in a freezer $\left(-70^{\circ} \mathrm{C}\right)$ until analysis.

As a control, total lipid of FCS was also extracted using the procedure of Bligh and Dyer (1959).

\section{Lipid analysis}

Fatty acid analysis. The total lipid extracts from oocytes and FCS were evaporated to dryness under a stream of nitrogen, and treated with $5 \%(\mathrm{v} / \mathrm{v}) \mathrm{HCl}$ in methanol in sealed vials for $2 \mathrm{~h}$ at $100^{\circ} \mathrm{C}$. After methanolysis, the fatty acid methyl esters were extracted using water-saturated hexane. The resultant fatty acid methyl esters were analysed by gas chromatography (G-3000, Hitachi; equipped with a hydrogen flame-ionization detector) and separated on a capillary column $(0.25 \mathrm{~mm}$ i.d. $\times 50 \mathrm{~m})$ of CP-Sil 88 . The column temperature was programmed from $160^{\circ} \mathrm{C}$ to $220^{\circ} \mathrm{C}$ at $2^{\circ} \mathrm{C} \mathrm{min}-1$. Injection and detection temperatures were maintained at $210^{\circ} \mathrm{C}$. The amount of each fatty acid was quantified through automatic calculation of peak area by a D-2500 chromato-integrator (Hitachi) to determine fatty acid composition.

Lipid content assay. Triglyceride, total cholesterol, phospholipids (phosphocholine-containing phospholipid) and non-esterified fatty acids from the oocytes were determined in fresh immature bovine oocytes and in aliquots of maturation medium containing FCS and polyvinyl alcohol using the triglyceride $\mathrm{E}$ test, total cholesterol E test, phospholipid C test, and the nonesterified fatty acid C test (Waco Pure Chemical Co., Osaka), respectively. Phosphocholine-containing phospholipid and non-esterified fatty acid contents were determined in frozen-thawed immature bovine oocytes to investigate the change in membrane phospholipids. For assays using triglyceride and non-esterified fatty acid kits, $100 \mu \mathrm{l}$ total lipid extracts from 100 oocytes was dried under a stream of nitrogen and reconstituted with $100 \mu \mathrm{l}$ isopropanol, and for assays using the cholesterol and phosphocholine-containing phospholipid kits, $100 \mu$ l total lipid extracts from 200 oocytes was reconstituted with $100 \mu \mathrm{l}$ isopropanol. A $20 \mu \mathrm{l}$ aliquot was taken for assay of triglyceride, total cholesterol and phosphocholinecontaining phospholipid (Youngs et al., 1994), and a $50 \mu \mathrm{l}$ aliquot was taken for assay of non-esterified fatty acid. All colorimetric assays were conducted in microplates and read at the appropriate wavelengths (600-700 nm for triglyceride, total cholesterol and phosphocholinecontaining phospholipid and 546-660 nm for non-esterified fatty acid) on a microtitre plate spectrophotometer (ELSIA Reader, International Reagent's Corp, Tokyo).

\section{Statistical analysis}

All comparisons were made between fresh and frozen-thawed immature oocytes. Data were analysed by one-way ANOVA and the Student's $t$ test. The same tests were used to compare immature and in vitro matured oocytes cultured in medium supplemented with FCS or polyvinyl alcohol.

\section{Results}

\section{Fatty acid composition}

The fatty acid composition in fresh and frozen-thawed immature bovine oocytes and in oocytes matured in vitro in medium containing FCS or polyvinyl alcohol is shown (Table 1). At least 22 fatty acids were detected, but only eight of these averaged $>2 \%(\mathrm{w} / \mathrm{w})$ of the total fatty acid content, and these were compared among experimental treatment groups. Palmitic acid (16:0) was the most abundant fatty acid in immature oocytes (A: 35\%; B: 36\%) and in in vitro matured oocytes cultured in medium supplemented with FCS (A: 36\%; B: 35\%) or polyvinyl alcohol (A: 33\%; B: 36\%). Palmitic acid content was not affected significantly by oocyte quality, freezing and thawing or by the degree of maturity of oocytes. However, the second most abundant fatty acid differed significantly: oleic (18:1) and stearic (18:0) acids occupied this position in A and B ranked oocytes, respectively (fresh and frozenthawed immature and in vitro matured oocytes). In addition, there were significant differences $(P<0.05)$ between $\mathrm{A}$ and $B$ ranked fresh immature oocytes in the proportions of linoleic (18:2; A: $6.5 \pm 0.3 ; \mathrm{B}: 4.6 \pm 0.3)$ and arachidonic (20:4; A: $4.4 \pm 0.3 ; \mathrm{B}: 2.3 \pm 0.4$ ) acids. In contrast, regardless of ranking, in vitro matured oocytes showed significant decreases $(P<0.05)$ in the proportions of linoleic and arachidonic acids. Similarly, significant decreases in the proportion of arachidonic acid were observed in both A and B ranked frozen-thawed oocytes (A: $2.4 \pm 0.5, \mathrm{~B}: 1.7 \pm 0.2$ ) compared with fresh immature oocytes. There was no significant difference in the fatty acid composition between in vitro matured oocytes cultured in the medium supplemented with FCS or polyvinyl alcohol. However, there was a tendency towards higher concentrations of oleic and stearic acids in in vitro matured oocytes cultured in medium containing FCS.

Linoleic acid was the most abundant fatty acid (37.6 \pm $0.4 \%$ ) and palmitic and stearic acids were the second and third most abundant.

\section{Lipid content}

Triglyceride was the most abundant lipid class in both immature and in vitro matured oocytes in medium containing FCS and polyvinyl alcohol (Table 3). The triglyceride content in immature oocytes (57.6 pmol per oocyte) decreased after $24 \mathrm{~h}$ of maturation (FCS: $36.6 \mathrm{pmol}$ per oocyte; polyvinyl alcohol: 27.7 pmol per oocyte). Thus, the decrease in triglyceride varied depending on the presence of FCS in the culture medium. The total cholesterol content (16.3 pmol per oocyte) was not altered after maturation in the presence of FCS (15.1 pmol per 


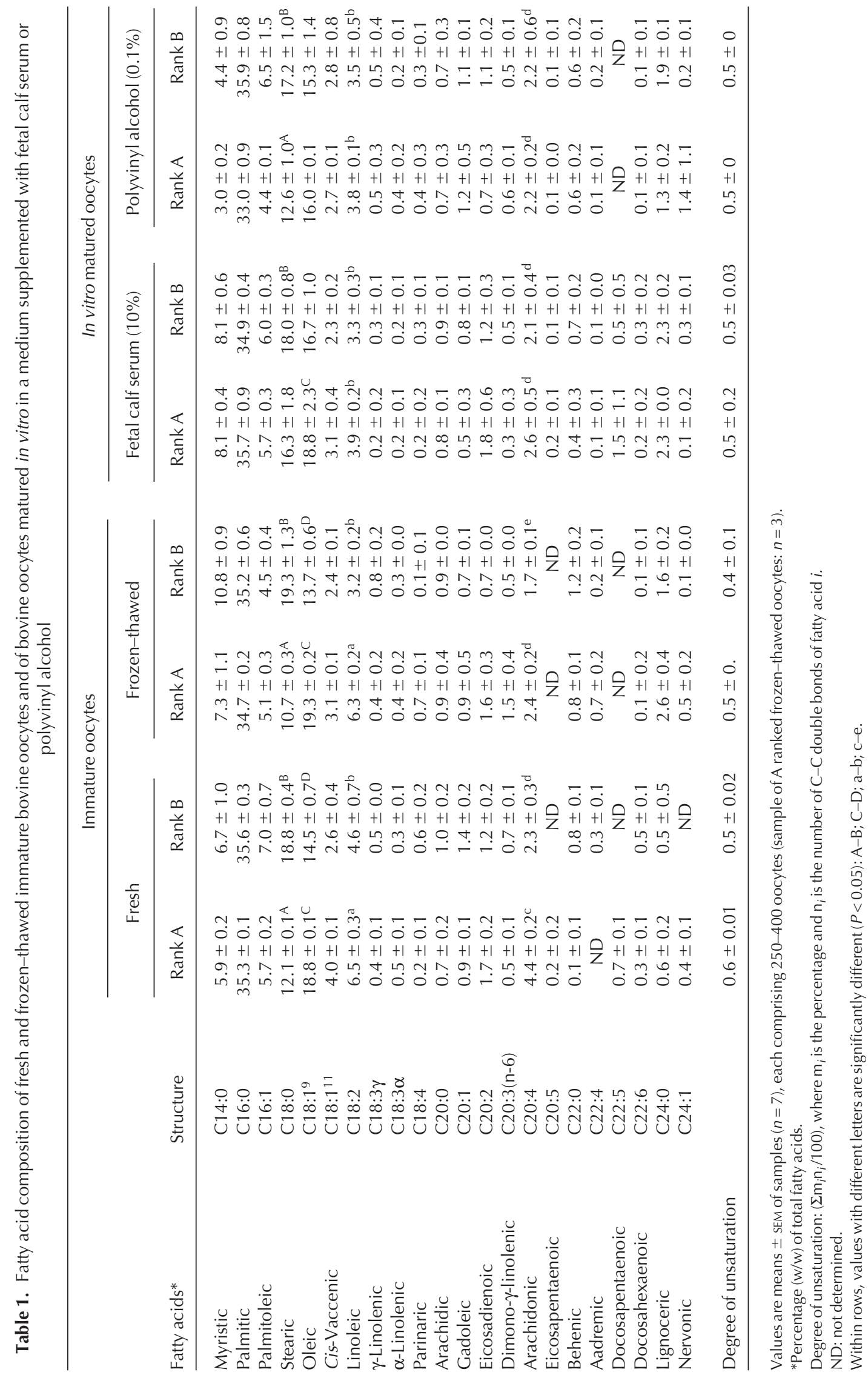


Table 2. Fatty acid composition of fetal calf serum used in the in vitro maturation medium

\begin{tabular}{llr}
\hline Fatty acids & Structure & $\begin{array}{r}\text { Percentage of } \\
\text { total fatty acid }\end{array}$ \\
\hline Palmitic & $\mathrm{C} 16: 0$ & $14.5 \pm 1.2$ \\
Palmitoleic & $\mathrm{C} 16: 1$ & $6.3 \pm 1.2$ \\
Stearic & $\mathrm{C} 18: 0$ & $14.1 \pm 2.6$ \\
Oleic & $\mathrm{C} 18: 19$ & $7.4 \pm 0.7$ \\
Linoleic & $\mathrm{C} 18: 2$ & $37.6 \pm 0.4$ \\
$\gamma$-Linolenic & $\mathrm{C} 18: 3 \gamma$ & $9.4 \pm 0.2$ \\
Parinaric & $\mathrm{C} 18: 4$ & $0.7 \pm 0.2$ \\
Arachidic & $\mathrm{C} 20: 0$ & $1.9 \pm 0.2$ \\
Dimono- $\gamma$-linolenic & $\mathrm{C} 20: 3(\mathrm{n}-6)$ & $2.6 \pm 0.2$ \\
Arachidonic & $\mathrm{C} 20: 4$ & $2.1 \pm 0.1$ \\
Eicosapentaenoic & $\mathrm{C} 20: 5$ & $1.6 \pm 0.4$ \\
\hline
\end{tabular}

Values are means \pm SEM of three replicates and are expressed as a percentage of the total fatty acid composition.

oocyte). In contrast, oocytes matured in medium containing polyvinyl alcohol had significantly $(P<0.01)$ lower total cholesterol content (9.2 pmol per oocyte). There were no differences in phospholipid and non-esterified fatty acid concentrations between immature and in vitro matured oocytes.

There was a significant $(P<0.05)$ decrease in both phosphocholine-containing phospholipid (15.8 pmol per oocyte and $10.6 \mathrm{pmol}$ per oocyte) and non-esterified fatty acid ( 11 pmol per oocyte and 4.1 pmol per oocyte) concentrations between fresh and frozen-thawed immature bovine oocytes.

\section{Discussion}

The results of this study provide the first detailed data on the changes in lipid and fatty acid composition in fresh and frozen-thawed immature, and in vitro matured, bovine oocytes cultured in media containing FCS or polyvinyl alcohol. In addition, differences in fatty acid composition were observed between morphologically distinct groups of bovine oocytes.
In mammals, storage lipids tend to be predominantly saturated and monosaturated fatty acids (Gurr and Harwood, 1991). Jeffcoat (1979) reported that the high concentrations of palmitic and oleic acids were found in oocyte neutral lipids, which were also required as substrates for fatty acid elongation and desaturation. In the present study, the analysis of fatty acid composition in all A ranked oocytes showed that palmitic (16:0), oleic (18:1) and stearic (18:0) acids were the first, second and third most abundant fatty acids, respectively. This finding was similar to those of studies in bovine (Zeron et al., 1999a; McEvoy et al., 2000) and pig oocytes (Homa et al., 1986; McEvoy et al., 1997). In contrast, Sata et al. (1999) showed that myristic acid (14:0) was the most abundant fatty acid in bovine embryos. Sata et al. (1999) indicated that the differences in fatty acid composition could be attributed to species differences and the different ages or breeds of cow used in the experiment. The present study demonstrates a difference in fatty acid composition of $\mathrm{A}$ and $\mathrm{B}$ ranked oocytes and indicates that the appearance of the ooplasm may reflect its lipid and fatty acid content.

Lower concentrations of oleic, linoleic and arachidonic acids were observed in B ranked oocytes compared with those of $A$ ranked oocytes. However, the lower concentrations of linoleic and arachidonic acids observed in B ranked oocytes were similar to those concentrations in in vitro matured oocytes regardless of rank. It is possible to relate these findings to those of other studies (Kruip et al., 1983; de Loos et al., 1989). de Loos et al. (1989) observed that bovine oocytes in categories 3 and 4 had larger lipid droplets than those in oocytes in categories 1 and 2, and that oocytes in categories 3 and 4 had maturation-like organelle arrangements. However, there was no significant difference in the ability of oocytes in categories 1,2 and 3 to undergo maturation in vitro, whereas oocytes in category 4 had a significantly diminished ability to mature in vitro (de Loos et al., 1989). There was also no significant difference in the in vitro maturation rates between $\mathrm{A}$ and $\mathrm{B}$ ranked oocytes in the present study (data not shown). Thus, it is possible to conclude that the different fatty acid composition in each ranked oocyte did not directly influence maturation, although fatty acid composition may be important for

Table 3. Lipid contents (pmol per oocyte) of fresh and frozen-thawed immature bovine oocytes and of bovine oocytes matured in vitro in a medium supplemented with fetal calf serum (FCS) or polyvinyl alcohol

\begin{tabular}{|c|c|c|c|c|}
\hline \multirow[b]{2}{*}{ Lipid class } & \multicolumn{2}{|c|}{ Immature oocytes } & \multicolumn{2}{|c|}{ In vitro matured oocytes } \\
\hline & Fresh & Frozen-thawed & FCS $(10 \%)$ & Polyvinyl alcohol $(0.1 \%)$ \\
\hline Triglyceride & $57.6 \pm 1.2^{\mathrm{a}}$ & ND & $36.6 \pm 1.2^{b}$ & $27.7 \pm 1.0^{\mathrm{c}}$ \\
\hline Total cholesterol & $16.3 \pm 1.3^{d}$ & ND & $15.1 \pm 1.1^{d}$ & $9.2 \pm 0.5^{\mathrm{e}}$ \\
\hline Phospholipid & $15.6 \pm 1.0^{f}$ & $10.5 \pm 0.9 \mathrm{~g}$ & $13.4 \pm 1.1^{f}$ & $12.1 \pm 0.7^{f}$ \\
\hline Non-esterified fatty acid & $11.0 \pm 1.2^{\mathrm{h}}$ & $5.5 \pm 0.3^{i}$ & $13.5 \pm 1.4^{\mathrm{h}}$ & $10.3 \pm 1.5^{\mathrm{h}}$ \\
\hline
\end{tabular}

Values are means \pm SEM of five replicate samples of 100-200 oocytes in 100-200 $\mu$ l.

ND: not determined.

Within rows, values with different letters are significantly different: $P<0.01$ : a-c, d-e; $P<0.05$ : $\mathrm{f}-\mathrm{g}$, h-i. 
oocyte competence and for the differences in fertilization and developmental potential.

Homa and Brown (1992) reported that a critical relative concentration of linoleic acid in follicular fluid may be important in regulating meiotic arrest of bovine oocytes at the germinal vesicle stage. In addition, Murakami et al. (1986) reported that linoleic acid stimulates protein kinase C, which plays a significant role in cell growth and differentiation (Nishizuka, 1988). In the present study, significantly lower $(P<0.05)$ amounts of linoleic and arachidonic acids were observed in the in vitro matured oocytes than in the immature oocytes. This difference is probably a result of the decrease in these acids during in vitro maturation as linoleic acid cannot be synthesized in animal cells. However, there was no significant difference in the concentrations of linoleic and arachidonic acids between oocytes matured in the media supplemented with FCS and those matured in media supplemented with polyvinyl alcohol. This finding indicates that the fatty acids in FCS did not affect the change in fatty acid composition in oocytes during in vitro maturation, although the FCS had a high concentration of linoleic acid.

Several studies have reported a positive effect of linoleic acid on cryopreservation of bovine embryos (Hochi et al., 1999a; Tominaga et al., 2000). It has been suggested that the concentration of polyunsaturated fatty acid in immature bovine oocytes may affect chilling sensitivity (Zeron et al., 1999b; McEvoy et al., 2000). In the present study, there was no significant difference in the survival rate between A ranked $(76.8 \%)$ and B ranked (67.2\%) frozen-thawed immature oocytes. However, arachidonic acid content and the concentration of unsaturated fatty acids were decreased in both $\mathrm{A}$ and $\mathrm{B}$ ranked frozen-thawed immature oocytes. Our unpublished data (J. Y. Kim, M. Kinoshita, M. Ohnishi and Y. Fukui) in another study observed that degenerated frozen-thawed oocytes, which usually are not selected, showed significantly lower concentrations of arachidonic acid $(0.8 \pm 0.2 ; \mathrm{w} / \mathrm{w})$. These lower concentrations may be due to the oxidative damage of oocyte membrane lipids during and after freezing and thawing. Arachidonic acid can be synthesized from linoleic acid, and oocytes may benefit by storing the more versatile precursor preferentially, which is at lower risk of free radical damage (McEvoy et al., 2000). Hochi et al. (1999b) found that a high linoleic acid concentration had a detrimental effect on cryopreservation of bovine embryos. Preliminary studies and the results of the present study indicate that the positive effects of linoleic acid may be dependent on the fatty acid components in an oocyte.

The contents of both phosphocholine-containing phospholipid and non-esterified fatty acid in the frozenthawed immature oocytes were lower than those in the fresh immature oocytes. This difference may reflect a loss of phospholipid in oocyte membranes. The loss of phosphocholine-containing phospholipid may increase the cholesterol:phospholipid ratio, leading to a decrease in membrane fluidity and reduced ability to tolerate low temperature (Anash and Buckland, 1994). In the present study, because the loss of phosphocholine-containing phospholipid occurred without an increase in non-esterified fatty acid, it can be postulated that phosphocholinecontaining phospholipid was metabolized to phosphatidic acids rather than deacylated to the water-soluble form. Fuku et al. (1995) speculated that the possible change of phosphocholine-containing phospholipid to phosphatidic acids may disrupt the oocyte membrane. It has been suggested that changes in lipid content of bovine oocytes may be associated with changes in the sensitivity of intracellular organelles to low temperature (Diez et al., 1996; Otoi et al., 1997). Therefore, the reduction in arachidonic acid content and the loss of phospholipid in frozen-thawed oocytes, as observed in the present study, may be the main reasons for the low survival and development rates after freezing and thawing procedures in other studies (Lim et al., 1992; Parks and Ruffing, 1992; Van Blerkum and Davis, 1994). However, it has not been established whether the membrane damage that occurred as a result of the freezing and thawing procedure resulted from the decrease of phosphocholine-containing phospholipid content per se or from the conversion of phosphocholine-containing phospholipid to phosphatidic acids. Further studies on oxidative damage of membranes in frozen-thawed oocytes are required to confirm these observations.

The fatty acid composition of cells grown in lipid-free media differs from that of cells grown in media containing serum (Geyer et al., 1961). Cells grown in a serumcontaining medium have a fatty acid profile that reflects the composition of the serum (Geyer et al., 1961; Boyle and Ludwig, 1962). Spector (1972) demonstrated that cells readily take up fatty acids, phospholipids and triglycerides from culture medium containing serum. In the present study, there was no significant difference in fatty acid composition between in vitro matured oocytes in the media containing FCS and those in the media containing polyvinyl alcohol. However, in vitro matured oocytes in the media supplemented with FCS tended to have higher concentrations of stearic (18:0) and oleic (18:1) acids regardless of ranking. The triglyceride and the total cholesterol contents in in vitro matured oocytes cultured in the medium containing FCS were significantly $(P<0.05)$ higher than those of in vitro matured oocytes cultured in the medium containing polyvinyl alcohol. These findings indicate that lipids and fatty acids may be incorporated into the oocyte cytoplasm during in vitro maturation.

Several studies have reported that triglyceride is the major lipid component of bovine and pig oocytes (Homa et al., 1986; McEvoy et al., 1997, 2000). Similarly, triglyceride was also found to be the most abundant lipid in immature and in vitro matured bovine oocytes in the present study. However, in the present study, the triglyceride content in in vitro matured oocytes was significantly $(P<0.01)$ lower than that of immature oocytes. This finding is in agreement with the study of Ferguson and Leese (1999), who reported 
that the triglyceride content of bovine oocytes before in vitro maturation was $59 \pm 1.37 \mathrm{ng}$ and decreased to $46 \pm 0.85 \mathrm{ng}$ after in vitro maturation. These findings and preliminary studies indicate that triglyceride can be used as an energy source for in vitro maturation of oocytes. However, as the triglyceride content in in vitro matured oocytes cultured in the media containing FCS was higher than that of in vitro matured oocytes cultured in the media containing polyvinyl alcohol, there is still a great need to understand the mechanisms involved in lipid nutrition and mechanisms for transfer of lipids from supplemented medium into oocytes. Further research on lipid biochemistry in bovine oocytes, including lipid peroxidation and endogenous lipid metabolism in the membrane, is required to determine the factors that influence in vitro maturation and the sensitivity to cryopreservation. By altering the effects of these factors before or during cryopreservation, improved results may be obtained after freezing and thawing of oocytes.

In conclusion, the present study indicates that the fatty acid composition of bovine oocytes is relevant to oocyte competence. Thus, oocyte selection for in vitro maturation and the cryopreservation tolerance of immature bovine oocytes may eventually be evaluated predictably by analysis of fatty acid composition. In addition, the protection of fatty acid and lipid components may play a vital role in the prevention of the damage currently related to cryopreservation and in the improvement of in vitro maturation systems for bovine oocytes.

The authors wish to thank J. G. Thompson for reading the manuscript and for his valuable criticism. Finally, the authors would like to thank $M$. Tachibana for his technical assistance in freezing and thawing of bovine oocytes.

\section{References}

Abe H, Yamasita S, Itoh T, Satoh T and Hoshi H (1999) Ultrastructure of bovine embryos developed from in vitro matured and fertilized oocytes: comparative morphological evaluation of embryos cultured either in serum-free medium or in serum-supplemented medium Molecular Reproduction and Development 53 325-335

Al Hasani S and Diedrich K (1995) Oocyte storage. In Gamete - The Oocyte pp 376-395 Eds JG Grudzinskas and JL Yovich. Cambridge University Press, Cambridge

Anash GA and Buckland RB (1994) Genetic variation in fowl semen cholesterol and phospholipid levels, and the relationships of these lipids with fertility of frozen-thawed and fresh semen Poultry Science $\mathbf{6 1}$ 623-637

Arav A, Zeron Y, Leslie SB, Behboodi E, Anderson GB and Crowe JH (1996) Phase transition temperature and chilling sensitivity of bovine oocytes Cryobiology 33 589-599

Asada M, Horii M, Mogoe T, Fukui Y, Ishkawa $\mathbf{H}$ and Ohsumi $\mathbf{S}$ (2000) In vitro maturation and ultrastructural observation of cryopreserved minke whale (Balaenoptera acutorostrata) follicular oocytes Biology of Reproduction 62 253-259

Bligh EG and Dyer WJ (1959) A rapid method of total lipid extraction and purification Canadian Journal of Biochemistry and Physiology 37 911-917

Boyle JJ and Ludwig EH (1962) Analysis of fatty acids of continuously cultured mammalian cells by gas-liquid chromatography Nature (London) $196893-894$ de Loos F, Van Vliet C, van Maurik P and Kruip TAM (1989) Morphology of immature bovine oocytes Gamete Research 24 197-204

Diez C, Le Bourhis D, Heyman Y and Renard JP (1996) Effect of partial lipid removal from in vitro produced bovine zygotes on further development in vitro and on the freezing tolerance of blastocysts Theriogenology 45 166 (Abstract)

Dobrinsky JR (1996) Cellular approach to cryopreservation of embryos Theriogenology 45 17-26

Ferguson EM and Leese HJ (1999) Triglyceride content of bovine oocytes and early embryos Journal of Reproduction and Fertility 116 373-378

Fuku E, Xia L and Downey BR (1995) Ultrastructure changes in bovine oocytes cryopreserved by vitrification Cryobiology 32 139-156

Geyer RP, Bennett A and Rohr A (1961) Fatty acids of the triglycerides and phospholipids of HeLa cells and strain L fibroblasts Journal of Lipid Research 3 80-83

Gurr MI and Harward JL (1991) The nature of lipids and their place in living things. In Lipid Biochemistry 4th Edn pp 4-9 Eds MI Gurr and JL Harward. St Edmundsbury Press, UK

Hochi S, Kanamori A, Sug'wa K, Kimura K and Hanada A (1999a) Effect of linoleic acid-albumin in the IVM and IVF media on survival of frozen-thawed pronuclear bovine zygotes Journal of Mammalian Ova Research 16 19-22

Hochi S, Kimura K and Hanada A (1999b) Effect of linoleic acid-albumin in the culture medium on freezing sensitivity of in vitro produced bovine morulae Theriogenology 52 497-504

Homa ST and Brown CA (1992) Changes in linoleic acid during follicular development and inhibition of spontaneous breakdown of germinal vesicles in cumulus-free bovine oocytes Journal of Reproduction and Fertility 94 153-160

Homa ST, Racowsky C and McGaughey RW (1986) Lipid analysis of immature pig oocytes Journal of Reproduction and Fertility 77 425-434

Jeffcoat (1979) The biosynthesis of unsaturated fatty acids and its control in mammalian liver Essays in Biochemistry 15 1-36

Kruip TAM, Cran DG, Van Beneden TH and Dieleman SJ (1983) Structural changes in bovine oocytes during final maturation in vivo. Gamete Research 8 29-47

Lee ES and Fukui Y (1995) Effect of various growth factors in a defined culture medium on in vitro development of bovine embryos matured and fertilized in vitro. Theriogenology 44 71-83

Leibo SP, Pollard JW and Martino A (1995) Chilling and freezing sensitivity of 'reassembled' in vitro derived bovine embryos Theriogenology $\mathbf{4 3}$ 265 (Abstract)

Lim JM, Fukui Y and Ono H (1992) Developmental competence of bovine oocytes frozen at various maturation stages followed by in vitro maturation and fertilization Theriogenology 37 351-361

McEvoy TG, Coull GD, Speake BK, Staines ME and Broadbent PJ (1997) Estimation of lipid and fatty acid composition of zona-intact pig oocytes Journal of Reproduction and Fertility Abstract Series 2010

McEvoy TG, Coull GD, Broadbent PJ, Hutchinson JSM and Speake BK (2000) Fatty acid composition of lipids in immature cattle, pig and sheep oocytes with intact zona pellucida Journal of Reproduction and Fertility 118 163-170

Murakami K, Chan SY and Routtenberg A (1986) Protein kinase C activation by cis-fatty acid in the absence of $\mathrm{Ca}^{2+}$ and phospholipids Journal of Biological Chemistry $26115424-15429$

Nishizuka Y (1988) The molecular heterogeneity of protein kinase $C$ and its implications for cellular regulation Nature 334 661-665

Otoi T, Yamamoto K, Koyama N, Tachikawa S, Murakami M, Kikkawa Y and Suzuki T (1997) Cryopreservation of mature bovine oocytes following centrifugation treatment Cryobiology 34 36-41

Parks JE and Ruffing NA (1992) Factors affecting low temperature survival of mammalian oocytes Theriogenology 37 59-73

Sata R, Tsujii H, Abe H, Yamashita S and Hoshi H (1999) Fatty acid composition of bovine embryos cultured in serum-free and serumcontaining medium during early embryonic development Journal of Reproduction and Development 45 97-103

Schmidt M, Hyttel P, Greve T and Avery B (1993) Ultrastructure of frozen-thawed bovine in vitro matured oocytes Theriogenology 39304 (Abstract) 
Spector AA (1972) Fatty acid, glyceride and phospholipid metabolism. In Growth, Nutrition and Metabolism of Cells in Culture pp 257-296 Eds GH Rothblat and VJ Cristofalo. Academic Press, New York

Thompson JG, Gardner AP, Mcmilliam WH and Tervit HR (1995) Lamb birth weight is affected by culture system utilized during in vitro pre-elongation development of ovine embryos Biology of Reproduction 53 1385-1391

Tominaga K, Hamada Y, Yabuue T and Ariyoshi T (2000) Effect of linoleic acid-albumin on post-thaw survival of in vitro produced bovine embryos at the 16-cell stage Journal of Veterinary Medicine Science 62 465-467

Van Blerkum J and Davis PW (1994) Cytogenetic, cellular and developmental consequences of cryopreservation of immature and mature mouse and human oocytes Microscopy Research and Technique 27 165-193
Youngs CR, Knight TJ, Batt SM and Beitz DC (1994) Phospholipid, cholesterol, triacylglycerol and fatty acid composition of porcine blastocysts Theriogenology 41343 (Abstract)

Zeron Y, Arav A and Sklan D (1999a) Fatty acid composition in bovine immature oocytes Theriogenology 51311 (Abstract)

Zeron Y, Pearl M, Borochov A and Arav A (1999b) Kinetic and temporal factors influence chilling injury to germinal vehicle and mature bovine oocytes Cryobiology 38 35-42

Received 1 December 2000.

First decision 29 January 2001.

Accepted 19 March 2001. 